\title{
Estimation of kinetic energy harvesting potential for self- powered wearable devices with 67,000 participants from the UK Biobank
}

This paper was downloaded from TechRxiv (https://www.techrxiv.org).

\section{LICENSE}

CC BY 4.0

SUBMISSION DATE / POSTED DATE

04-06-2021 / 08-06-2021

\section{CITATION}

Beach, Christopher; Casson, Alex (2021): Estimation of kinetic energy harvesting potential for self-powered wearable devices with 67,000 participants from the UK Biobank. TechRxiv. Preprint.

https://doi.org/10.36227/techrxiv.14662713.v1

$\mathrm{DOI}$

10.36227/techrxiv.14662713.v1 


\title{
Estimation of kinetic energy harvesting potential for self-powered wearable devices with 67,000 participants from the UK Biobank
}

\author{
Christopher Beach, Member, IEEE and Alexander J. Casson, Senior Member, IEEE
}

\begin{abstract}
Energy harvesting from human motion can reduce reliance on battery recharging in wearable devices and lead to improved adherence. However, to date, studies estimating energy harvesting potential have largely focused on small scale, healthy, population groups in laboratory settings rather than free-living environments with population level participant numbers. Here, we present the largest scale investigation into energy harvesting potential by utilising the activity data collected in the UK Biobank from over 67,000 participants. This paper presents detailed stratification into how the day of the week and participant age affect harvesting potential, as well as how the presence of conditions (such as diabetes, which we investigate here), may affect the expected energy harvester output. We process accelerometery data using a kinetic energy harvester model to investigate power output at a high temporal resolution. Our results identify key differences between the times of day when the power is available and an inverse relationship between power output and participant age. We also identify that the presence of diabetes substantially reduces energy harvesting output, by over $21 \%$. The results presented highlight a key challenge in wearable energy harvesting: that wearable devices aim to monitor health and wellness, and energy harvesting aims to make devices more energy autonomous, but the presence of medical conditions may lead to substantially lower energy harvesting potential. The findings indicate how it is challenging to meet the required power budget to monitor diseases when energy autonomy is a goal.
\end{abstract}

Index Terms-Big data applications; energy harvesting; wearable computers

\section{INTRODUCTION}

$\mathbf{E}$ NERGY harvesting promises to reduce reliance on battery charging for wearable devices, either wholly or in part, by scavenging ambient energy from the environment to power the device. Reducing reliance on battery recharging is essential as battery charging remains one of the main barriers to adoption of wearables, where battery lifetime is typically limited to a few days [1]-[3]. Moreover, wearable devices

This work was supported by the UK Engineering and Physical Sciences Research Council grant number EP/N509565/1, EP/P010148/1 and the Doctoral Training Partnership with the University of Manchester

C. Beach and A. J. Casson are with the Department of Electrical and Electronic Engineering, University of Manchester, Manchester, UK (e-mail: christopher.beach@manchester.ac.uk, alex.casson@manchester.ac.uk).

Software used to perform the analysis in this manuscript is available from: DOI 10.48420/14720964. UK Biobank data utilised in the analysis in this publication is available by following the procedure described at http://www.ukbiobank.ac.uk/using-the-resource/. are often intended to benefit groups such as the elderly and those with cognitive impairments, meaning it is essential to make the wearable sensing automated and unobtrusive to the user [4], and energy harvesting is a key method to achieve this autonomy. Many different forms of energy harvesting are possible, for example using heat or motion as the energy input. For wearables, many energy harvesting papers (e.g. [5]-[7]) use kinetic energy harvesting which collects energy based upon the movement of a small proof mass.

While kinetic energy harvesting may be able to make wearable sensing free from user-intervention, research into kinetic energy harvesting has to date focused on small-scale laboratory studies rather than the large-scale free-living investigations that are required to allow accurate estimations of energy harvesting output and stratification by a range of factors including age, time of day, and the presence of diseases. To date, the majority of kinetic energy harvesting studies investigate power output from only one participant, with few investigating harvesting on over 10 participants [8]-[18]. Additionally, only a minority investigate energy harvesting in a free-living environment, rather than estimating output from periodic movements such as walking on a treadmill. These controlled experiments will overestimate harvesting potential against real-world usage, as the actual proportion of time spent undertaking periodic movements is relatively small. To our knowledge the largest study investigating energy harvesting in a free-living environment analyses 20 participants over 2 days [13].

In this paper we present a large-scale estimation of energy harvesting potential using data from the UK Biobank. We make use of data from $>67,000$ participants who wore an accelerometer device for a week. This dataset allows the first, to our knowledge, energy harvesting estimation analysis at a population level, allowing the spread of energy values between many different individuals in everyday life to be investigated. The results presented in this paper represent the largest energy harvesting study in number of participants, by three orders of magnitude, and over the longest duration (seven days) allowing weekday and weekend variances to be observed. Further, this is combined with the large amount of metadata available in the UK Biobank, enabling a large number of sub-analyses to be performed. This paper analyses when energy is available throughout the day and stratifies this by age, gender and season, which has not been possible in previous, much smaller scale, energy harvesting modelling analyses. This paper also investigates how the amount of collected energy is affected 
when participants are diagnosed with a long term condition, looking at the effect of diabetes on energy harvesting output. The results allow far more detailed insights into the practicality of using kinetic energy harvesters in real-world situations.

Our methods for estimating energy harvesting potential are based upon a widely used mass-spring-damper model [19] which takes measures of motion from a wrist worn accelerometer to estimate the movement of a proof mass and consequently the energy that could be collected by an energy harvester put in the same location. It builds upon our previous work optimising kinetic energy harvesters for wearables [20] where we demonstrated the optimum harvester parameters for various positioning on the body. Here, we utilise the model from [20] to analyse the output from non-repetitive movements in a free-living accelerometer dataset.

The remainder of this paper is structured as follows. In Section II we introduce the UK Biobank dataset used in this study, our inclusion criteria, the steps for preparing the raw data for our model, and the statistical methods used to compare differences in harvested power between different groups. In Section III we present the results, showing both the variation in harvested power against the time of day as well as the differences in power and total energy harvested for the different population groups. In Section IV we place the results in context, discussing the differences that were identified and whether the average power output seen here could power a typical wearable. In Section IV we also present the limitations of this work.

\section{METHODS}

\section{A. The UK Biobank Dataset}

The UK Biobank dataset is a large prospective study with over 500,000 participants in total, aged 40-69 years and recruited between 2006 and 2010 [21]. The UK Biobank collected a wide range of data from participants including biological samples (such as blood, urine and saliva), questionnaires on lifestyle, socio-demographic factors, cognitive function, family history, and medical history (including the prevalence of non-communicable diseases such as diabetes, chronic kidney disease and mental illnesses) [22]. After the initial recruitment of participants to the study, further data was collected from subsets of participants including blood pressure, electrocardiograms (ECG), hand grip strength, eye measurements, hearing tests, and arterial stiffness [22]. In addition, the physical activity of just over 100,000 participants was measured by inviting them to wear a wrist-worn accelerometer for 24-hours a day, for a week, at some point between 2013 and 2015 [23]. For a small number of participants $(2,500)$, repeated accelerometer measurements were undertaken over a year on a seasonal basis.

For these physical activity measurements,participants were asked to wear a 3-axis accelerometer (Axivity AX3) [24] on their dominant wrist. The sensor was designed to store data locally (rather than transmitting data in real-time) and was able to last for a least a week of continuous recording without requiring recharging. The device was designed to be comfortable and waterproof to allow for continuous 24hour monitoring without the need for sensor removal. Each participant's accelerometer was programmed to start recording at 10:00 two days after the device was due to arrive in the post. Further references to the UK Biobank in this paper refer specifically to the activity monitoring data rather than any of the other samples collected as part of the study.

The activity monitoring dataset available to this study contains data for 103,651 participants wearing the device for a week each, with participants aged between 43-78. Other studies in the literature using UK Biobank data contain slightly different numbers of participants, such as 101,516 participants in [25], 103,712 participants in [23] and 103,702 participants in [26]. The exact pool of participants available to different researchers varies as participants are allowed to remove their consent after data collection, as well as differing inclusion criteria. This research has been conducted using the UK Biobank Resource under Application Number 33693.

\section{B. Participant Selection from the UK Biobank}

Not all of the 103,651 participants who were sent an accelerometer successfully, or correctly, wore the device for the entire week. As a result, only a subset of participants were included in this analysis to ensure valid and complete data were present in all cases. Firstly, not all participants wore the accelerometer for the requested continuous 24-hours each day, with many wearing the device for a substantially shorter time. To counter this, the UK Biobank accelerometer expert group [23] recommends excluding participants who wore the device for less than a cumulative total of three days, and who did not wear the device during each one-hour period in a day. [23] identified this as generating activity values within $10 \%$ of the values calculated from participants who wore the device continuously, for their particular analysis. Using these criteria gives 96,600 participants, excluding less than $10 \%$ of the total dataset. However, for the participant analysis we undertake here, this criteria would skew the results towards representing when the device was worn rather than the fundamental amount of energy that could be collected at different times of the day. Rather than only selecting participants who wore the accelerometer for the full 24-hours each day (20,100 participants, $20 \%$ of the data), we opted to only include participants who wore the device for a minimum of 20 hours each day, giving over 70,000 participants and temporal coverage across most of the day. Participants who remained in our data selection with periods of non-wear (of up to four total hours per day) did not have these specific nonwear periods excluded from the analysis. Further participants were excluded as follows.

The AX3 accelerometer used in this study featured a USB port that was accessible to the participants, and numerous participants plugged the device into a computer when it was set to be recording. If a device was plugged in, recording would be paused and an interrupt would be logged. To account for these gaps in the data, participants who had large amounts of data missing ( $>1$ minute) were removed from the analysis $(n=300)$.

The data collection of physical activity from participants in the UK Biobank was carried out continuously in batches across 
multiple years, recording groups of participants at different times throughout the year. As a result, some recording sessions occurred during a daylight savings crossover. Depending on the crossover (entering or leaving daylight savings), this causes either multiple records of data for an hour, or an hour of data missing. While this crossover occurs during the night, when harvested energy potential is likely to be the lowest, only a small number of participants ( $n=4,543)$ were undergoing recording during a crossover, and therefore it was decided to remove all of the participants recorded during a daylight savings crossover in order to allow the power values from different times of day to be easily averaged together.

Participants in the UK Biobank were aged between 43 and 78 during accelerometry data collection (according to our age estimations-discussed later in Section II-C). However, only a small number $(n=153)$ were below the age of 45 , an amount considerably lower than in any other age group, and therefore all of these participants were removed from the analysis. Finally, any participants that had removed consent between the downloading of the dataset from the UK Biobank repository and our data analysis had to be removed $(n=15)$.

After these criteria, 67,024 participants were remaining, a number still substantially larger than the previous largest energy harvesting study on free-living data in the literature (20 participants) [13].

\section{Data Extraction and Stratification}

1) Accelerometer Data Extraction and Loading into Python: We downloaded the raw bulk accelerometer data from UK Biobank Data-Field 90001 in CWA (Continuous Wave Accelerometer) format, a compressed proprietary binary data format. The compressed dataset was approximately $20 \mathrm{~TB}$ in size, and we made use of high-performance computing facilities, the Computational Shared Facility 3 (CSF3) at the University of Manchester, to process this data. A complete analysis of the dataset took approximately two weeks to process across a large number of compute nodes.

For data pre-processing, we initially calibrated each participant's data to local gravity to account for variations in gravity in different locations (sensors are typically calibrated to set $1 \mathrm{~g}$ as the measurement of the acceleration due to gravity at their location of manufacture, but this does not necessarily correspond to the same value in the location where data recording takes place [27]). We then resampled the data to $100 \mathrm{~Hz}$ using linear interpolation, a process which is required as the AX3 accelerometer does not sample at precise intervals. The processing steps used here are based upon the protocol described by Doherty et al. [23] and their code available at [28]. Finally, each axis of the data was filtered with a sixthorder zero-phase high-pass Butterworth filter with a cutoff of $0.3 \mathrm{~Hz}$ to remove the gravity component. Although the gravity component is removed by low-pass filtering, we still correct for local gravity to remain consistent with pre-processing steps taken by other groups working on the UK Biobank accelerometer data. The data was processed in Python 3.7 using the Anaconda Distribution.

Initial analysis identified that at 10:00 on Monday, Wednesday, Thursday, Friday, and Saturday there was a large discon-

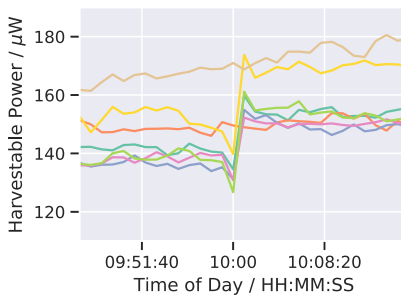

(a)

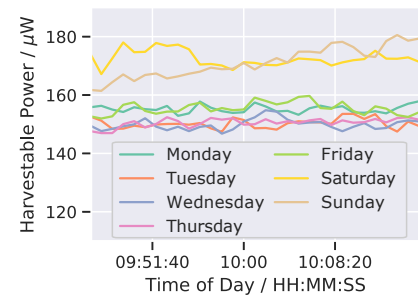

(b)
Fig. 1: Power output from the energy harvester model for each day of the week. (a) Harvesting output before removing data discontinuity, (b) Harvesting output after removing discontinuity.

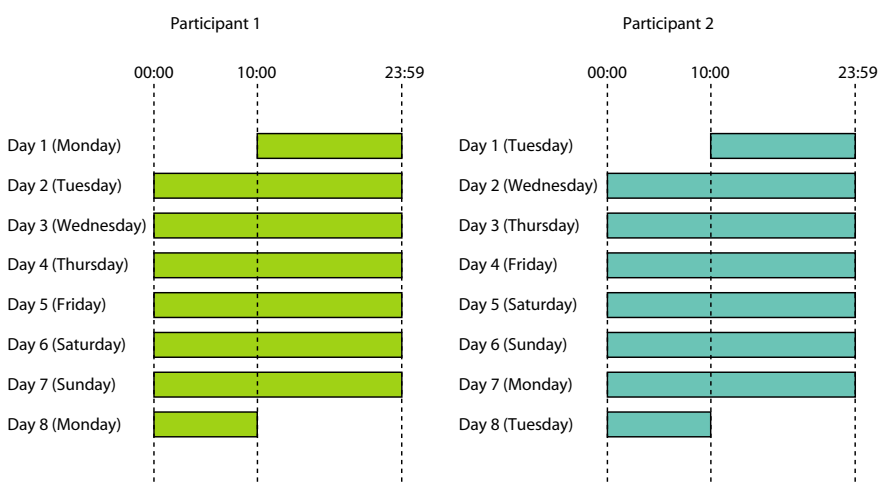

Fig. 2: Example of the recording routine for two participants in the UK Biobank.

tinuity in the estimated energy harvesting potential, where at 09:59 the power harvested was considerably lower than the power at 10:01, which is demonstrated in Fig. 1a. This effect was not was not present on Tuesday or Sunday. We also noted how no participants started the experiment on a Sunday and only a small number $(<2 \%$ of participants) started the experiment on a Tuesday, suggesting that the discontinuity is an artefact of the process of starting or stopping the experiment. As the data collection began on 10:00 on the participant's particular day, we hypothesised that participants may have removed their wrist band early before the data collection had finished, which caused this discontinuity. Participants were told the data collection would last 7 days, they, therefore, assumed data collection had ended by day eight, while in actuality the device was still recording, and would not finish until 10:00 on the eighth day of data collection. This protocol is illustrated in Fig. 2. In UK Biobank Resource 141141, the participant instructions detail that they should wear the device 'continuously until the morning of [End Date] when it will switch itself off automatically', rather than explicitly specifying to only remove it after 10:00 when the device stopped recording.

To verify this, the energy harvester output between 08:0010:00 on each day of the week was calculated (excluding Sunday as no participants started on this day), stratifying participants into the particular day of the week they started the experiment on. The first/last day for each participant was removed, with the resultant data shown in Fig. 1b. Here it 
can be seen that the discontinuity has been removed, and the data is continuous between 09:59-10:01. To correct for this, for all of the subsequent analyses in this paper the day of the week of the first day of wear was removed. Due to the recording starting at 10:00, this effectively results in the first and last day of wear, which sums to 24-hours, being removed for all participants. To our knowledge this discrepancy has not been previously reported or identified, as most studies have calculated their results at substantially lower temporal resolution than we do so this effect is not noticeable. (Due to the size of the data set, studies (e.g. [23], [29]) plot one hour epochs of the accelerometry data, whereas we summarise results on a minute-by-minute basis.) Considering this limitation is important to create valid analyses and thus are important for future researchers to be aware of.

2) Age Estimation: To protect participant anonymity, the UK Biobank does not make available to researchers the exact date of birth of participants, instead only providing their year and month of birth. The exact date on which the accelerometer recording was started is known and made available. To estimate the participant's age from these fields, we assumed that each participant was born on the $15^{\text {th }}$ of the month, making the estimate of the participant's age potentially out by a maximum of 16 days. Further, if the participant's birthday estimate occurs during the week-long data collection period, this is not split up as two separate participants, instead just taking their age as the age they were when they started data collection. This increases the error in age estimation to 22 days if their birthday was at the start of the data collection period. This slight fuzziness in grouping participants is accepted as a limitation of the study. Participants are also grouped into seasons of the year during data collection, calculated by taking the month when they started the data collection as the season, categorising March-May as spring, June-August as summer, September-November as autumn, and December-February as winter. Again, if the season changed during the data collection, the record was not split into two parts, but categorised as the season the data collection started in. The sex of participants was identified using UK Biobank Data-Field 31.

3) Diabetes Diagnosis: After analysing all participants together, we also investigate the effects of the presence of a long term condition on energy harvesting potential. This requires the generation of a dataset with this condition and a matched control dataset to reference against. The UK Biobank dataset contains participants who reported having a diagnosis of diabetes during the initial questionnaire stage of the study, which is of interest due to the potential to use wearables to monitor conditions of diabetes, such as diabetic foot ulcers [30]. The UK Biobank Data-Field 2443 asked participants if they have ever had diabetes diagnosed by a doctor. This gave a total of 2,389 participants with diabetes as the experimental group (using the same exclusion criteria detailed in Section II-B).

To generate a set of control group of participants with no diagnosis of diabetes, theoretically all the remaining participants in the UK Biobank could be used. However, this would result in an unbalanced comparison as the control group would have an order of magnitude more participants [31]. Instead, a control group was randomly selected to match the number of participants in the diabetic group, matching them on both the number in each of the four age groups in our study, and the number of each sex within those age groups. Further, for generating a matched control data set, it is important to note that Data-Field 2443 on diabetes diagnosis was collected at recruitment to the study (for some participants as early as 2006). Some participants started the activity monitoring stage of the study as late as 2015, a potential maximum difference of 9 years. Based on an estimated $0.17 \%$ of the general population being diagnosed with diabetes each year (based off 300 people being diagnosed per day with diabetes in 2011 [32]), it was assumed that around $1 \%$ of the control group could have developed diabetes by the time they carried out the accelerometry data collection. This is an amount which we assume will have a negligible effect on the results presented but should be noted as a potential limitation.

Given the discrepancy between the date of metadata collection and accelerometer data collection, we identified the time lag between these two elements of data. The time lag was $2087 \pm 406$ days (5.7 years) and $2064 \pm 402$ days (5.7 years) between metadata and accelerometer data collection for the control (declaring they had no diagnosis of diabetes) and the group with diabetes (declaring they had a diagnosis of diabetes) respectively. As it is relatively uncommon for diabetes to go into remission [33], even when well controlled, it was assumed that in the diabetes group, diabetes was still present during data collection. Additionally, given the random selection of control group participants, this group may also have a variety of other diseases despite not having diabetes. This was not controlled for as it was assumed that most younger participants had no other conditions while many of the older group had a variety of comorbidities, which would be representative of the actual population that the energy harvesting output is to be compared against.

\section{Dataset Statistics}

Full statistical information on the analysed participants in the subset of the UK Biobank used in this paper is shown in Table I, and the subset of participants with diabetes (and associated control participants) is shown in Table II. Values of participants age are mean \pm SD (standard deviation). Participants were grouped into four age groups, which were not all of equal sizes in order to match the age groupings used in [23].

\section{E. Harvesting Methods}

Each of the accelerometry files was processed with the steps that we have previously described [20]. This process briefly compromises of modelling the kinetic energy harvester as a second-order system with a proof mass, $m$, damper, $b$, and spring constant, $k$. The movement of the proof mass over time, $z(t)$, is described by (1),

$$
z(t)=\mathcal{L}^{-1}\left\{\frac{d(s)}{m s^{2}+b s+k}\right\} .
$$

where $d(s)$ is the external displacement acting on the frame of the harvester, $s$ is the Laplace variable, and $\mathcal{L}^{-1}$ the 
TABLE I: Number of participants in each of the analysed groups from the full UK Biobank accelerometer records.

\begin{tabular}{|c|c|c|c|c|}
\hline \multirow[b]{2}{*}{ Stratification } & & \multicolumn{3}{|c|}{ Number of Participants } \\
\hline & & Female & Male & Total \\
\hline \multirow{4}{*}{ Age Groups } & $45-54$ & 7749 & 5085 & 12834 \\
\hline & $55-64$ & 13812 & 9387 & 23199 \\
\hline & $65-74$ & 15208 & 14128 & 29336 \\
\hline & $74-78$ & 743 & 912 & 1655 \\
\hline \multirow{4}{*}{ Season } & Spring & 8397 & 6451 & 14848 \\
\hline & Summer & 10334 & 8093 & 18427 \\
\hline & Autumn & 10651 & 8368 & 19019 \\
\hline & Winter & 8130 & 6600 & 14730 \\
\hline \multicolumn{2}{|l|}{ Total } & 37512 & 29512 & 67024 \\
\hline \multicolumn{2}{|c|}{ Age (Mean \pm SD) } & $61.8 \pm 7.6$ & $63.2 \pm 7.7$ & $62.4 \pm 7.6$ \\
\hline
\end{tabular}

TABLE II: Number of participants in the sub-set with diabetes and the control group used for comparison.

\begin{tabular}{|c|c|c|}
\hline \multirow[b]{2}{*}{ Sex } & \multicolumn{2}{|c|}{ Number of Participants } \\
\hline & Control & Diabetes \\
\hline Female & 892 & 892 \\
\hline Male & 1497 & 1497 \\
\hline Age $($ Mean \pm SD) & $64.7 \pm 7.0$ & $65.1 \pm 7.0$ \\
\hline
\end{tabular}

inverse Laplace transform. The proof-mass movement is then converted into power at each time point, $P(t)$,using (2),

$$
P(t)=b\left(\frac{d z(t)}{d t}\right)^{2}=b(v(t))^{2}
$$

where $v(t)$ is the velocity of the proof-mass. We used the recommended parameters from [20] that we previously identified for a harvester placed on the wrist with a participant walking at $100 \mathrm{steps} / \mathrm{min}$ (taken as the lower end of a brisk walking pace [34]), and a physical harvester size of $Z_{L}=$ $50 \mathrm{~mm}$. These parameters were $m=0.11 \mathrm{~g}, b=0.07 \mathrm{~kg} / \mathrm{s}$, $k=12.15 \mathrm{~kg} / \mathrm{s}^{2}$, which give a resonance frequency, f $f_{r}$, of $0.71 \mathrm{~Hz}$ and $Q$ of 0.55 for the second order system described by (1). As in [20] we calculate the maximum theoretical power output of an energy harvester, taking the efficiency as $100 \%$ for the majority of the analyses presented here. This allows us to investigate the maximum theoretical values of energy collectable in free-living environments, which can then be scaled as desired to a practical energy harvester implementation. We also compare the output of our energy harvester model to the power consumption of some commercial wearable devices. Here we assumed the efficiency of our harvester is $20 \%$, based on similar efficiency levels found in practical harvesters [35].

The data output from the harvester power model was then downsampled from $100 \mathrm{~Hz}$ to $1 / 60 \mathrm{~Hz}$, equivalent to 1 sample per minute, to reduce the quantity of data and enable further analysis with manageable file sizes. The accelerometers record acceleration in 3-axes, while our energy harvesting model requires data of a single dimension. Only one axis of the data was analysed, again taking the axis that produced the largest amount of power for the majority of participants, which was the $\mathrm{x}$-axis (which aligns with the forward swinging motion of the arm). We add that in our model developed in [20], we noted that in physical realisations of an energy harvester the proof mass cannot travel further than its physical end-stop limits. In [20] we did not limit the movement of the proof mass in simulation, choosing instead to limit the values of $m$, $b$ and $k$ so that the proof-mass did not displace further than the end-stop limits of the frame, for the activities that we asked participants to undertake. Given the free-living dataset used here, it is possible that extremely large participant movements may cause the theoretical proof-mass to exceed the limits of the harvester frame. This is taken as a limitation of this work, but it is assumed that this only occurs in a minority of cases and does not have a substantial impact on the results presented.

\section{F. Selection of Data}

Both the power output throughout the day, and differences in average power harvested per groups of participants, were analysed. To compare the average power harvested between groups, the period from 07:00 to 20:00 was analysed, defined here as daytime hours, chosen as the point where the harvested power level is over $50 \%$ of the maximum, rounded to the nearest hour. This is to ensure that differences between groups are not biased by the very small amount of power that is harvested during the night (i.e. when most participants were asleep), under-reporting the differences between the groups. We also expect the maximum allowed non-wear time (four hours total per day) to occur during the night period.

\section{G. Statistical Methods}

To analyse the differences between groups statistical methods were applied using the SciPy library in Python. The Student's t-test [36] was used for comparisons of two groups with the significance level $(\alpha)$ set as $p<0.05$. For comparisons between multiple groups, the one-way analysis of variance (ANOVA) [37] was calculated again with $\alpha$ set as $p<0.05$. An ANOVA identifies if one or more sample distributions are not equal, but not where these differences occur. The ANOVA is also considered as robust to non-normal data [38]. To identify where (if any) differences occur, post-hoc tests were run to identify which groups had differences. Here the Bonferroni correction was used to account for multiple comparisons. Multiple comparisons are made between the four age groups and four seasons, of which there are a total of six comparisons in each, so the adjusted $\alpha$ value is corrected to $p<0.0083$. Effect sizes were calculated using Cohen's d with small, medium and large effect sizes of $>0.2,>0.5$ and $>0.8$ respectively [39]. 95\% confidence intervals (CIs) were also calculated for the variations in power output throughout the day.

\section{H. Analysis Overview}

Following the data processing steps described above, we then present the results of the analysis by stratifying it in several ways:

Firstly, the variations in power output throughout the 24hour period of the day are analysed. This gives new insights into how the harvested power varies as the day progresses, 
compared to other studies looking at free-living energy harvesting which have presented the average power available throughout the day rather than how this varies with time [13], or over shorter periods up to 11 hours in [8]. Within our analysis, the power output across the day is stratified by:

- Variations by day of the week

- Variations by age group

- Variations by presence of diabetes

Secondly, the variations in power output during daytime hours (07:00-20:00) are presented, allowing comparison between a range of groups that have not previously been considered, including the presence of a diabetes diagnosis and how the energy harvesting potential varies at different times of a year. For this analysis, participants are stratified by:

- Variations by sex

- Variations by age

- Variations by season

- Variations by presence of diabetes

Finally, we consider the percentage of a day where the energy harvester power output is above set power thresholds, for the entire dataset. This analysis allows designers to identify the likelihood that a given energy budget will be met when using a harvester in an average population.

\section{Results}

\section{A. Energy Harvester Output Throughout the Day}

1) Variations by Day of the Week: First, the variation in power harvested across the day for each day of the week is shown in Fig. 3, with the shaded areas showing 95\% confidence intervals. The confidence intervals are relatively narrow and are therefore covered by the mean trend line, suggesting that the mean offers a good estimate of the true data. In Fig. 3 it can be seen that each weekday (MondayFriday) follows a similar pattern. Between the hours of 00:0006:00 the average power harvested is $\langle 25 \mu \mathrm{W}$, while the vast majority of people are asleep. After 06:00 the harvested power begins a steady increase, reaching the maximum value of around $150 \mu \mathrm{W}$ at 09:00. The average power output begins a steady decline until around 13:30, where it remains around $120 \mu \mathrm{W}$ until 19:00. Beyond this, the power follows a generally decreasing trend with large peaks, around $10 \mu \mathrm{W}$ high. Here it can be seen that these peaks often fall on the hour (for example at 21:00 and 22:00), corresponding to larger amounts of activity taking place on the hour. There are also multiple smaller peaks between 18:00-23:00 corresponding to increasing activity taking place not only on the hour but also on the half, quarter past and quarter to the hour.

The weekend shows different patterns to the weekdays. Saturday shows a later time of day where the harvested power begins to ramp up after the night, starting to increase at around an hour later (07:00 compared to 06:00 in the week), the data on Sunday shows this later increase in the morning as well, but starts to increase slightly later again (by around 15 minutes). The weekend has a higher maximum power, reaching over $180 \mu \mathrm{W}$ in the morning, and remaining higher than the weekdays until around 17:00 by at least a $10 \mu \mathrm{W}$ margin. Into the evening (18:00-21:00), the weekend has lower power output than the weekdays. In the early hours of the morning at the weekend (00:00-02:00) the harvested power is higher than during the week. The peaks of power seen on the hour between 18:00-22:00 are much less prominent on Saturday but are still relatively visible on Sunday. There are also small differences in the total energy harvested for each day of the week, with Sunday having the smallest total energy. The total energy harvested for each day was $7.09 \mathrm{~J}$, $7.10 \mathrm{~J}, 7.09 \mathrm{~J}, 7.10 \mathrm{~J}, 7.05 \mathrm{~J}, 7.22 \mathrm{~J}$ and $6.95 \mathrm{~J}$ for Monday, Tuesday, Wednesday, Thursday, Friday, Saturday and Sunday respectively.

2) Variations by Age Group: Fig. 4 shows the variations in harvested power throughout the day for each of the four age groups detailed in Section II, again with shaded areas showing 95\% confidence intervals. Between the hours of 00:00-05:00 there are very few differences between the groups, with all groups harvesting $\leq 25 \mu \mathrm{W}$. As in Fig. 3, the power begins to increase after 06:00, with the youngest age group (45-54) being the first to increase, followed by the second oldest (55$64)$, with the oldest two groups (65-74 and 75-79) following a similar pattern to each other until 10:00. For a brief period in the morning (09:00-10:30) three groups, 45-54, 55-64, and $65-74$, display similar levels of power at just over $150 \mu \mathrm{W}$. For the majority of the day, the power output is inversely proportional to the age of the participant.

Comparing with Fig. 3, where the power output decreased as the afternoon progressed, the 45-54 age group sees an increase in power output between 16:45-18:45. From 19:15 onwards all groups see a sharp decrease in power output with time, with small differences between groups after 23:00. As in Fig. 3, there are peaks of power occurring on the hour from 19:00 22:00, which are prominent in each of the age groups. The total energy harvested across the a 24-hour period for each of the age groups decreases with age, with values of $8.21 \mathrm{~J}$, $7.40 \mathrm{~J}, 6.43 \mathrm{~J}$ and $5.42 \mathrm{~J}$ for the $45-54,55-64,65-74$ and 74-78 age groups respectively.

3) Variations by Presence of a Disease: The data in Fig. 5 shows the variations in harvester power throughout the day for the group of participants with diabetes and the age/sex matched control group used as a reference. From 00:0006:00 the diabetic group generates more power compared to the control group, with small differences of around $100 \mu \mathrm{W}$. After 07:00 this crosses over and the power output from the control group is larger than the diabetic group by $30 \mu \mathrm{W}$ between 10:00-18:00. Into the evening the differences between the two groups decrease with little difference from around 21:00 onwards. Again, the peaks of power that occur on the hour in Fig. 3 and Fig. 4 are visible in both of the groups, with both showing very similar trends, just offset by different amounts throughout the day. There is a difference of over $1 \mathrm{~J}$ between the total energy harvested across the day between the control participants and participants with diabetes, with values of $6.93 \mathrm{~J}$ and $5.56 \mathrm{~J}$ respectively. Further investigation into the differences in power harvested between these two groups during daytime hours of the day is given in Section III-B. 


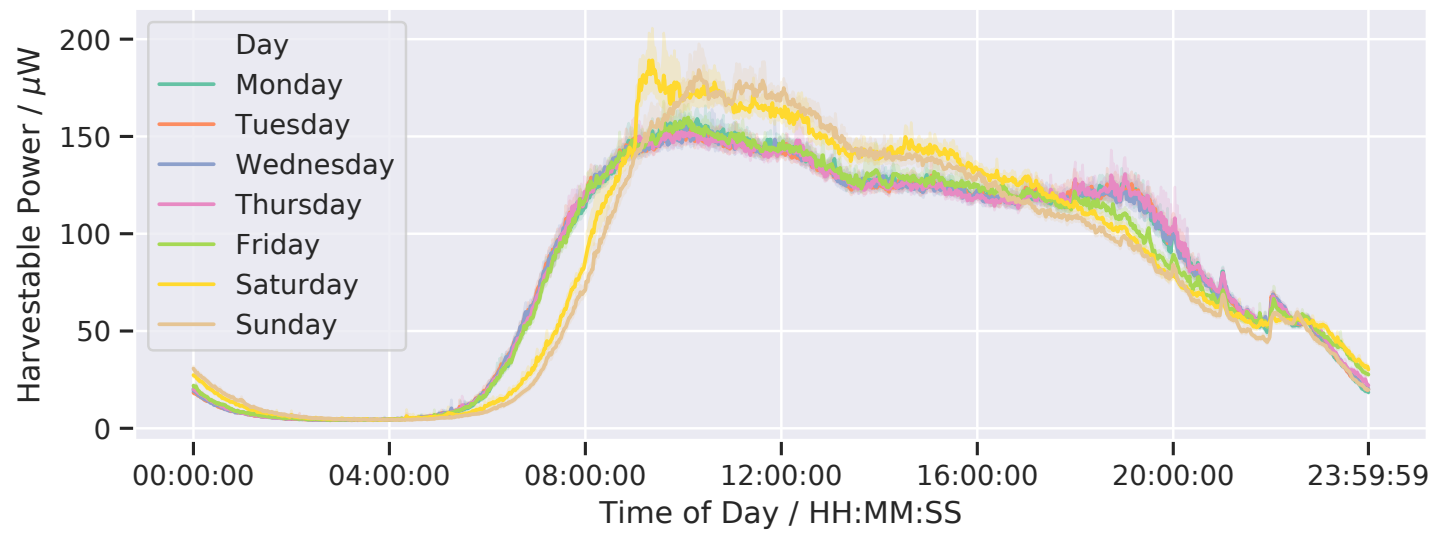

Fig. 3: Average power harvested per day across all participants by day of the week. Shaded areas show 95\% confidence intervals.

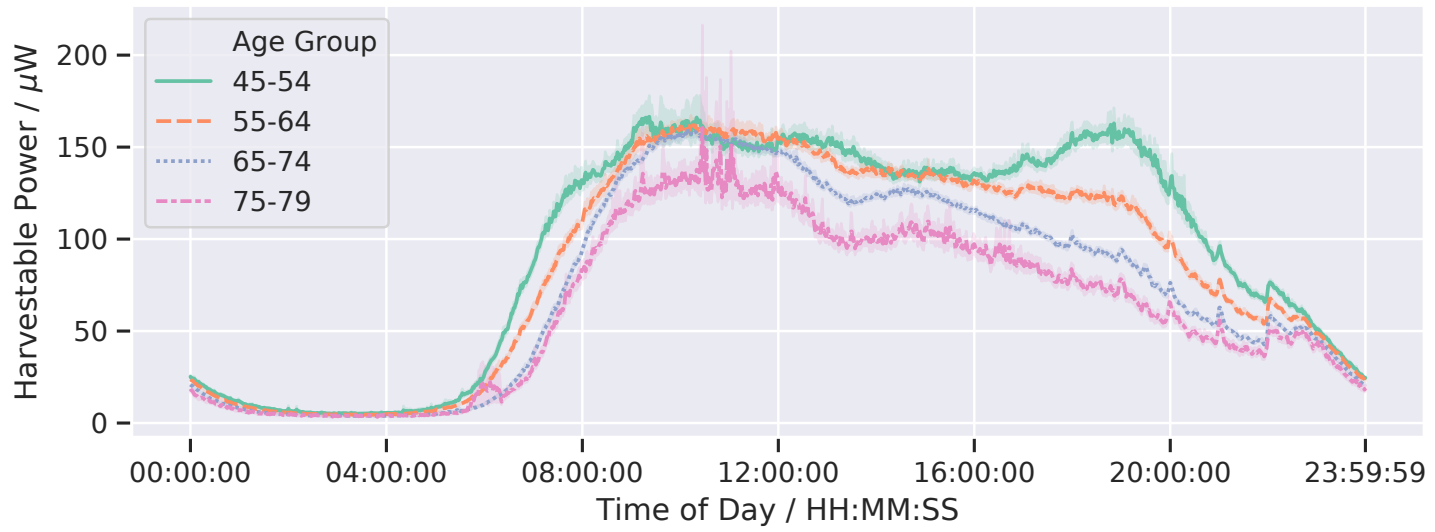

Fig. 4: Average power harvested across the day for each of the age groups of participants. Shaded areas show 95\% confidence intervals.

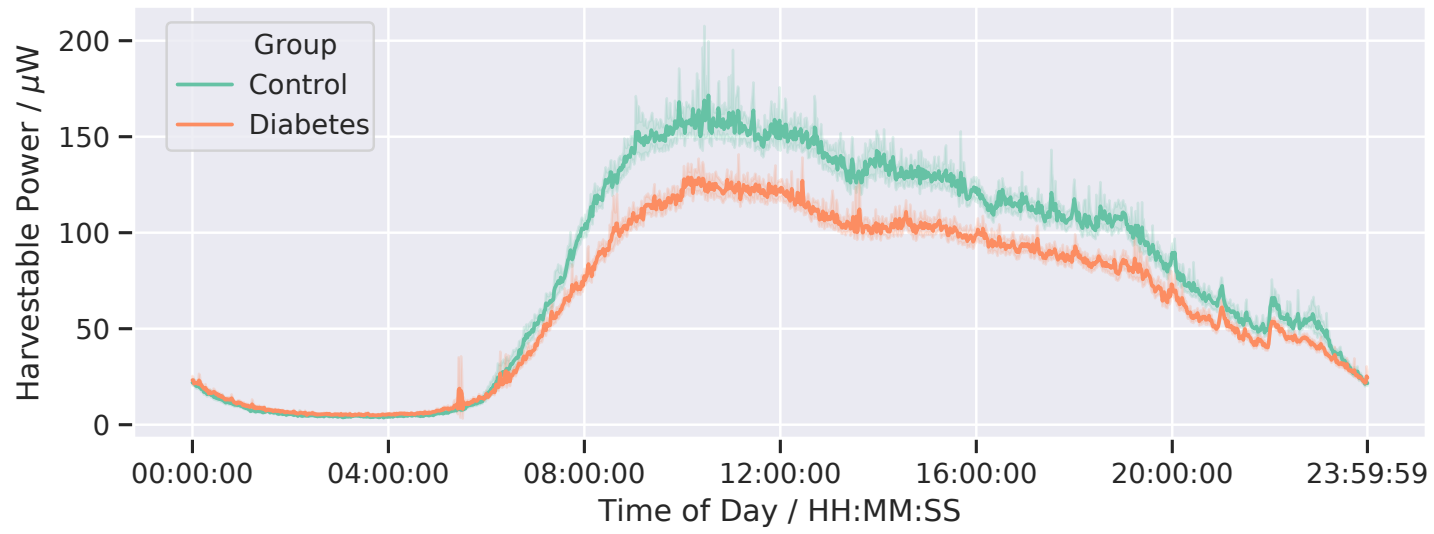

Fig. 5: Average power harvested across the day for control participants and participants with diabetes. Shaded areas show 95\% confidence intervals. 


\section{B. Average Power Harvested During Daytime Hours}

1) Variations by Sex: Female participants generated a marginally larger average harvested power during daytime hours compared to males, with medians of $118 \mu \mathrm{W}$ for female participants compared with $113 \mu \mathrm{W}$ for male participants. Interquartile ranges (IQRs) were $58.9 \mu \mathrm{W}$ and $66.2 \mu \mathrm{W}$ for females and males respectively. Differences were not significant $(p=0.104)$.

2) Variations by Age: During daytime hours differences between each of the age groups again followed the pattern shown for the majority of the day in Fig. 3, where power was inversely proportional to age. The median power harvested for each of the groups was $128 \mu \mathrm{W}, 121 \mu \mathrm{W}, 109 \mu \mathrm{W}$ and $91.5 \mu \mathrm{W}$ for the $45-54,55-64,65-74$ and 75-79 age groups respectively. IQRs were $68.0 \mu \mathrm{W}, 62.1 \mu \mathrm{W}, 58.0 \mu \mathrm{W}$ and $49.3 \mu \mathrm{W}$ for each age group respectively. Significant differences between groups were found $(p<0.000001)$. The post-hoc tests identified significant differences between all combinations of age groups $(p<0.000001)$, all differences between age groups corresponded to a small effect, except for differences between groups 45-54 and 55-64, which corresponded to no effect. No effect size highlights how these differences may be statistically significant (as is often the case with very large sample sizes [40]), but the actual differences are very small.

3) Variations by Season: Differences in average harvested power during daytime hours were small between each of the four seasons. Median harvester power was $120 \mu \mathrm{W}, 118 \mu \mathrm{W}$, $115 \mu \mathrm{W}$ and $112 \mu \mathrm{W}$ in Spring, Summer, Autumn and Winter respectively. IQRs were $65.0 \mu \mathrm{W}, 62.5 \mu \mathrm{W}, 61.3 \mu \mathrm{W}$ and $59.6 \mu \mathrm{W}$ respectively. Significant differences between groups were found $(p<0.000001)$. The post-hoc tests found significant differences between all combinations of seasons (except for Spring-Summer), and in all these cases these differences corresponded to no effect.

4) Variations by Presence of a Disease: We consider again the impact of a diabetes on harvesting power, this time comparing average power during daytime hours. Here, the median power was $114 \mu \mathrm{W}$ and $90 \mu \mathrm{W}$ for control and participants with diabetes respectively, a difference of over $21 \%$. IQRs were $63.1 \mu \mathrm{W}$ and $55.6 \mu \mathrm{W}$ respectively. Differences were again significant $(p<0.000001)$ with a small effect size.

\section{Time Spent in Harvesting Zones}

Finally, the percentage of the day where the energy harvester is collecting power over specific energy thresholds is considered. The analysis includes all 67,024 participants from this study, and allow designers insight into the required duty cycling of their device. If a device has a specific known average power consumption, the amount of the day the device will be able to operate at for that power level can be found. In Fig. 6, the x-axis shows a range of minimum harvesting levels and the $y$-axis shows the percentage of the day that the energy harvester output is above this threshold.

From Fig. 6, it can be seen that if the theoretical device had an average power consumption of $1 \mu \mathrm{W}$, the device would be able to operate for over $60 \%$ of the day, for an average

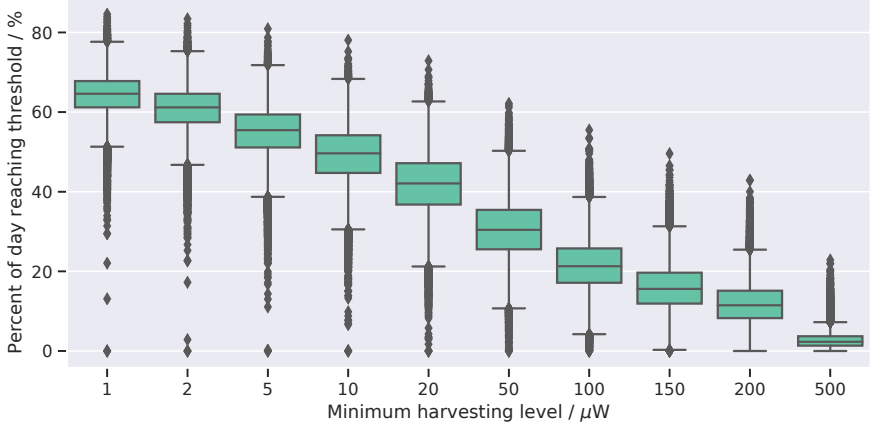

Fig. 6: Percentage of the day that minimum harvesting levels are reached. The central line in the box plot represents the median percentage of participants reaching that threshold, denotes outliers.

user. The data in Fig. 6 is presented as a box-plot to allow visualisation of how the levels vary across participants, by showing the levels of the $25 \%$ and $75 \%$ quartiles of the data, the limits of the data range and outliers. From the box plot we can also gain insights into how many participants will reach each threshold. With Fig. 6 we can see that for a power consumption of $1 \mu \mathrm{W}, 50 \%$ of participants will be able to operate the device at this level for $60-65 \%$ of the day. The data in Fig. 6 considers all participants in the analysed dataset and does not stratify them based on age, season, sex, day of week or prevalence of a disease, allowing the data here to closely represent the average population. Further, the results here do not consider the effect of any energy storage element paired with the energy harvester and the associated changes this could have on the power availability from losses or flattening of the power demand. Importantly, outliers are present for all of the minimum energy harvesting levels, which will always be the case in real-world scenarios. Even with a small power budget of around $1-2 \mu \mathrm{W}$ for a particular device, there will be some users who we cannot get this power from. It is important that designs or usage patterns reflect this.

\section{DISCUSSION}

\section{A. Implications of Results}

1) Variations in Power Across the Day: We demonstrated in Fig. 3 how, on average across participants, large peaks of power are available on the hour (with smaller peaks at the half-hour, quarter past and quarter to the hour) in the afternoon and evening. These peaks likely correspond to large groups of people moving at a similar time, and this being fixed to events that happen on the hour. The cause for this may be potentially driven by broadcast television, where programmes are often set to start and finish on the hour. Therefore, as a programme is starting or finishing a large number of the participants are moving to watch television or getting up after the programme has finished. This effect is widely known for affecting the UK's National Grid [41], where it is known as TV pickup; as large proportions of the population operate devices during commercial breaks in transmission, causing high electrical demand and noticeable strain on the UK electricity generation 
network. This finding introduces the possibility of scheduling power intensive device operations with when there is large amounts of power available. Previously, we have discussed the possibility of timing operations with the power that is available from each footstep [42].

In the population level data in this paper, the peaks of power from individual footsteps are not visible, but the larger-scale movements are visible (typically on a minute by minute basis rather than a few second basis as would be the case with timing with footsteps). The peaks of power highlighted in Fig. 3 increase in height by around $10 \mu \mathrm{W}$ over the baseline. This potentially could be used in opportunistic transmission [43] as the device can predict that there is a high probability of there being more power available on the hour and wait to do its power-hungry operations (such as wireless transmission [44]) at this point. Comparing this figure to an average of $135 \mu \mathrm{W}$ over $3.5 \mathrm{~ms}$ that is required to transmit a single byte of data on the Bluetooth Low Energy (BLE) Nordic Semiconductor nRF52832 device [45] (assuming $V_{c c}=3.0 \mathrm{~V}$ ), the energy required for full opportunistic transmissions is some way from being directly realizable. Fitting BLE packets directly into these peaks is out of reach by around 10 times, but given that these peaks over power last for multiple minutes, and it only takes $2.5 \mathrm{~ms}$ to send a byte of data, it is possible that combining with an energy storage element could allow devices to have sufficient power to transmit using this known peak. However, this effect may potentially diminish or decrease in prominence in the future as younger groups move away from broadcast television and towards streaming services [46], where the effect of the TV pickup seen by the UK's National Grid is already starting to decrease in prominence [47].

The data shown in the line plots in Fig. 3, Fig. 4 and Fig. 5 all show a similar shape, representing a typical pattern as someone active from approximately 09:00-19:00, sleeping between approximately 23:00-07:00, and fairly sedentary in the evenings. There do not seem to be sufficient participants who work night shifts in the UK Biobank to show any bimodal patterns in the overall average. There is not a clear second group of people who work shifts and sleep at odd patterns or during the day. This would be seen as a bimodal distribution around the mean, which is not present in the shown confidence intervals in these plots. The UK Biobank does record participants who work shifts and night shifts (under UK-Biobank Data-Fields 826 and 3426 respectively). Further work could involve an investigation into how this changes the pattern of power generation and how it introduces uncertainties into the estimations.

2) Meeting Wearable Power Requirements: During daytime hours the average power harvested was around $105 \mu \mathrm{W}$. Using the approximation of $20 \%$ conversion efficiency as in [20], this gives around $21 \mu \mathrm{W}$ of available power during the day. Comparing this to the average power of some popular wearables using the values given in [20], we can compare the harvesting power levels against the power consumption of popular commercial devices. For example, the Fitbit Charge 3 consumes on average $1.6 \mathrm{~mW}$, a figure two orders of magnitude larger than the average harvester power output. Even ignoring conversion efficiency, there is still a substantial gap between the power required and that which can be generated. This suggests that fully autonomous operation in free-living environments may not be feasible with current kinetic energy harvester approaches, and some form of battery backup will always be required.

3) Variations Between Groups: Patterns in age differences shown in Fig. 4 are not necessarily what might be expected. Given the youngest group in this study is 45-54, whom are all of working age and therefore likely to be at work, with most UK workers spending their time stationary [48] particularly for office workers [49], [50], it could be expected that this group would generate the least power, as they spend a majority of the time sitting at a desk with little periodic movement and therefore little energy generation. This trend is not present, with all age groups up to 75 producing similar levels of energy in the morning (between approximately 8:0012:00). In the afternoon the energy produced decreases as the age group increases. We start to see more potentially expected differences in the late afternoon and evening where the 45-54 group finishes work and commute home, perhaps undertaking activities with family or exercise while the older group undertakes less activity. Further, the peaks in the evening on the hour are likely attributed to the fact that older people are more likely to watch broadcast TV than the younger group [51].

4) Variations by Presence of a Disease: The group of participants with diabetes showed significant differences in energy harvesting output compared to the healthy control group. The diabetes group harvested $21 \%$ less power, a statistically significant difference. These differences were also visualised across the time series in Fig. 5, where we could see how differences in power output were small at night and largest during the morning. These differences are possibly due to those with diabetes being less active than the general population as they struggle to carry out physical activity [52]. This highlights a key challenge in wearable energy harvesting-wearable devices aim to monitor people's health and wellness, and energy harvesting aims to make devices energy-autonomous and fit-and-forget-but in populations with a disease there is potentially less power available to harvest and therefore it is harder to meet the required power budget and do the desired health and wellness monitoring. Future work would be beneficial to investigate how the trend seen for people with diabetes applies to other conditions and people with comorbidities.

\section{B. Limitations}

The results presented in this paper are for a fixed harvester configuration, using the optimum parameters for a participant walking at $100 \mathrm{steps} /$ minute sized at $Z_{L}=50 \mathrm{~mm}$ as determined in [20]. The walking cadence that this harvester is optimised for may not correspond to the optimum harvester parameters for a harvester on a free-living group (rather than a group in laboratory conditions walking on a treadmill), and it is possible that other combinations could improve power output. However, even with different harvester configurations we would expect to see similar trends across the groups 
analysed in this paper, with a much greater impact on the absolute values of power. To identify optimum values, the harvester parameters would have to be swept on a large number of the UK Biobank records, which would be very computationally intensive (the records in this manuscript are 7 days long, compared with $40 \mathrm{~s}$ in [20]). Alternatively, records could be analysed to find their dominant frequency and a harvester designed with a resonant frequency $f_{r}$ centred on this. However, as was shown in [20] this does not always produce the optimum combination. Such design approaches still only optimise the harvester based on periodic walking movements. Other activities may have a drastically different frequency spectrum to walking and optimising for these spectrums may improve power output. This may however also increase variation between participants, as some may generate lots of harvestable energy from non-periodic movements, but others very little. Given the scale of data and results, inevitability this paper has presented only one harvester configuration. The code is openly available with the details provided at the start of this paper, and the data openly available on application to the UK Biobank, to allow the investigation of other harvester configurations.

There are also some limitations in the data extraction methods used, including having to estimate the participant's age (which could be incorrect by up to 22 days). However, this is likely to have little impact on the results as changes in activity are not likely to occur as soon as participants cross an age threshold. Further, given the time-lag between metadata collection and data collection, participants in the comparison control group (in the diabetes analysis) could have received a diagnosis diabetes in the interim. We assumed this was a negligible number of people.

Datasets such as the UK Biobank are intended to represent the average population, but are known to be skewed by healthy volunteer bias. In particular in the UK Biobank, participants have been found to be more likely to be older, female, and to live in less socioeconomically deprived areas than the general UK population [53]. While this may have an impact on the results presented here (particularly those in Fig. 6 where the data is intended to represent the average UK population), there are no other datasets available of the size and scale of the UK Biobank to generate an analysis such as the one presented in this paper. It provides an insight into the potential for using energy harvesting to power wearable devices in free-living environments at a scale that has not previously been possible.

\section{CONCLUSION}

We processed free-living accelerometer data from 67,024 UK Biobank participants to identify their energy harvesting potential and how this varies between different population groups. This work represents the largest energy harvesting study on free-living data, in terms of participant numbers, by three orders of magnitude. By utilising the UK Biobank we have been able to stratify energy harvester output by day of the week, age, sex, season and a presence of a disease (diabetes). These factors have not previously been investigated in energy harvesting studies as datasets were too small to allow detailed stratification. We have presented new insights into the times of day where power is available, highlighting how this varies between days of the week, where a maximum of $150 \mu \mathrm{W}$ was available around 09:00, and approximately $120 \mu \mathrm{W}$ until 19:00. This pattern was different on Saturday and Sunday, where the power ramp up was later, and a higher maximum power was available (approximately $180 \mu \mathrm{W}$ ). We have identified that there are distinct patterns in the data, such as $10 \mu \mathrm{W}$ peaks occurring on the hours in the evening, identifying how many in this population have bursts of activity around the hour. This effect has not been highlighted before as previous analysis of free-living accelerometer data has been at a substantially lower temporal resolution. Alongside this, we have also provided insights into how the power output varies throughout the day for different age groups. We identified significant differences in average power output between many groups, including age, season and presence of a disease, although in many cases these differences were either of a small effect size or no effect. The results presented in this paper enable detailed insights into energy harvesting potential across population groups that has not previously been possible.

\section{ACKNOWLEDGEMENTS}

The authors would like to acknowledge the assistance given by Research IT and the use of the Computational Shared Facility at The University of Manchester.

\section{REFERENCES}

[1] J. Williamson et al., "Data sensing and analysis: Challenges for wearables," in ASP-DAC, Chiba, Japan, Jan. 2015, pp. 136-141.

[2] M. Hassan et al., "Kinetic-powered health wearables: Challenges and opportunities," Computer, vol. 51, no. 9, pp. 64-74, 2018.

[3] J. D. Amor and C. J. James, "Setting the scene: Mobile and wearable technology for managing healthcare and wellbeing," in IEEE EMBC, Milan, Italy, Aug. 2015, pp. 7752-7755.

[4] X. Fafoutis et al., "Energy neutral activity monitoring: Wearables powered by smart inductive charging surfaces," in IEEE SECON, London, UK, Jun. 2016, pp. 1-9.

[5] P. D. Mitcheson et al., "Energy harvesting from human and machine motion for wireless electronic devices," Proc. IEEE, vol. 96, no. 9, pp. 1457-1486, 2008.

[6] C. Wei and X. Jing, "A comprehensive review on vibration energy harvesting: Modelling and realization," Renew. Sust. Energy Rev., vol. 74, pp. 1-18, 2017.

[7] H. Liu et al., "A comprehensive review on piezoelectric energy harvesting technology: Materials, mechanisms, and applications," Appl. Phys. Rev., vol. 5, no. 4, p. 041 306, 2018.

[8] M. Gorlatova et al., "Movers and shakers: Kinetic energy harvesting for the internet of things," IEEE J. Sel. Areas Commun., vol. 33, no. 8, pp. 1624-1639, 2015.

[9] N. G. Elvin and A. A. Elvin, "Vibrational energy harvesting from human gait," IEEE/ASME Trans. Mechatronics, vol. 18, no. 2, pp. 637-644, 2012.

[10] G. V. Merrett, H. Huang, and N. M. White, "Modeling the effect of orientation on human-powered inertial energy harvesters," IEEE Sens. J., vol. 15, no. 1, pp. 434-441, 2015.

[11] J.-P. Martin and Q. Li, "Overground vs. treadmill walking on biomechanical energy harvesting: An energetics and EMG study," Gait Posture, vol. 52, pp. 124-128, 2017. 
[12] W. Xu et al., "KEH-Gait: Using kinetic energy harvesting for gait-based user authentication systems," IEEE Trans. Mobile Comput., vol. 18, no. 1, pp. 139-152, 2019.

[13] S. Zhang and A. Seyedi, "Statistical models for harvested power from human motion," IEEE J. Sel. Areas Commun., vol. 33, no. 8, pp. 1667-1679, 2015.

[14] J.-P. Martin and Q. Li, "Design, model, and performance evaluation of a biomechanical energy harvesting backpack," Mech. Syst. Signal Process., vol. 134, p. 106318, 2019.

[15] Q. Li, M. Shepertycky, and J.-P. Martin, "Lower-limb driven energy harvesting backpack: Design, performance and energetics," in CCC, Hangzhou, China, Sep. 2015, pp. 7821-7826.

[16] J.-P. Martin and Q. Li, "Generating electricity while walking with a medial-lateral oscillating load carriage device," $R$. Soc. Open Sci., vol. 6, no. 7, p. 182 021, 2019.

[17] T. Xue et al., "System modeling, characterization, and design considerations for generators in commercial watches with application to energy harvesting for wearables," IEEE/ASME Trans. Mechatronics, vol. 23, no. 5, pp. 2515-2524, 2018.

[18] S. Zareei and J. D. Deng, "Energy harvesting modelling for self-powered fitness gadgets: A feasibility study," Int. J. Parallel Emergent. Distrib. Syst., vol. 34, no. 4, pp. 412-429, 2019.

[19] T. von Buren et al., "Optimization of inertial micropower generators for human walking motion," IEEE Sens. J., vol. 6, no. 1, pp. 28-38, 2006.

[20] C. Beach and A. J. Casson, "Inertial kinetic energy harvesters for wearables: The benefits of energy harvesting at the foot," IEEE Access, vol. 8, pp. 208 136-208 148, 2020.

[21] C. Sudlow et al., "UK Biobank: An open access resource for identifying the causes of a wide range of complex diseases of middle and old age," PLoS Med., vol. 12, no. 3, e1001779, 2015.

[22] C. Bycroft et al., "The UK Biobank resource with deep phenotyping and genomic data," Nature, vol. 562, no. 7726, pp. 203-209, 2018.

[23] A. Doherty et al., "Large scale population assessment of physical activity using wrist worn accelerometers: The UK Biobank study," PLoS One, vol. 12, no. 2, e0169649, 2017.

[24] Open Lab at Newcastle University. (2019). Open movement, [Online]. Available: https : / / github . com / digitalinteraction/openmovement.

[25] J. Firth et al., "The validity and value of self-reported physical activity and accelerometry in people with schizophrenia: A population-scale study of the UK Biobank," Schizophr. Bull., vol. 44, no. 6, pp. 1293-1300, 2018.

[26] E. Tikkanen, S. Gustafsson, and E. Ingelsson, "Associations of fitness, physical activity, strength, and genetic risk with cardiovascular disease: Longitudinal analyses in the UK Biobank study," Circulation, vol. 137, no. 24, pp. 2583-2591, 2018.

[27] V. T. Van Hees et al., "Autocalibration of accelerometer data for free-living physical activity assessment using local gravity and temperature: An evaluation on four continents," J. Appl. Physiol., vol. 117, no. 7, pp. 738-744, 2014.

[28] activityMonitoring. (2019). Biobankaccelerometeranalysis, [Online]. Available: https : / / github . com / activityMonitoring biobankAccelerometerAnalysis.

[29] M. Willetts et al., "Statistical machine learning of sleep and physical activity phenotypes from sensor data in 96,220 UK Biobank participants," Sci. Rep., vol. 8, no. 1, p. 7961, 2018.

[30] C. Beach et al., "Monitoring of dynamic plantar foot temperatures in diabetes with personalised 3D-printed wearables," Sensors, vol. 21, no. 5, p. 1717, 2021.

[31] T. Zebin, N. Peek, and A. J. Casson, "Physical activity based classification of serious mental illness group participants in the UK Biobank using ensemble dense neural networks," in IEEE EMBC, Berlin, Germany, Jul. 2019, pp. 1251-1254.
[32] Diabetes UK. (2016). Facts and stats - october 2016, [Online]. Available: https: / / www. diabetes.org.uk/ (visited on $04 / 29 / 2020$ ).

[33] L. McCombie et al., "Beating type 2 diabetes into remission," BMJ, vol. 358, j4030, 2017.

[34] C. Tudor-Locke et al., "How fast is fast enough? Walking cadence (steps/min) as a practical estimate of intensity in adults: A narrative review," Br. J. Sports Med., vol. 52, no. 12, pp. 776-788, 2018.

[35] D. Berdy, D. Valentino, and D. Peroulis, "Kinetic energy harvesting from human walking and running using a magnetic levitation energy harvester,' Sens. Actuator A Phys., vol. 222, pp. 262-271, 2015.

[36] Student, "The probable error of a mean," Biometrika, vol. 6, no. 1, pp. 1-25, 1908

[37] R. Armstrong, S. Slade, and F. Eperjesi, "An introduction to analysis of variance (ANOVA) with special reference to data from clinical experiments in optometry," Ophthalmic Physiol. Opt., vol. 20, no. 3, pp. 235-241, 2000.

[38] E. Schmider et al., "Is it really robust?" Methodology, vol. 6, no. 4, pp. 147-151, 2010.

[39] J. Cohen, Statistical Power Analysis for the Behavioral Sciences. Routledge, 1988.

[40] G. M. Sullivan and R. Feinn, "Using effect size—or why the p value is not enough," J. Grad. Med. Educ., vol. 4, no. 3, pp. 279-282, 2012.

[41] D. W. Bunn and J. P. Seigal, "Forecasting the effects of television programming upon electricity loads," J. Oper. Res. Soc., vol. 34, no. 1, pp. 17-25, 1983.

[42] C. Beach, P. R. Green, and A. J. Casson, "Optimizing energy harvesting for foot based wearable sensors," in IEEE EMBC, Honolulu, HI, USA, Jul. 2018, pp. 1185-1188.

[43] S. S. Gupta and N. B. Mehta, "Revisiting effectiveness of energy conserving opportunistic transmission schemes in energy harvesting wireless sensor networks," IEEE Trans. Commun., vol. 67, no. 4, pp. 2968-2980, 2019.

[44] C. Beach, E. Balaban, and A. J. Casson, "Edge algorithms for wearables: An overview of a truly multi-disciplinary problem," in Wearable Sensors, E. Sazonov, Ed., Second Edition, Oxford: Academic Press, 2021, pp. 379-414.

[45] Nordic Semiconductor. (2019). nRF52 Online Power Profiler, [Online]. Available: https : / / devzone. nordicsemi . $\mathrm{com} / \mathrm{nordic} /$ power.

[46] Mintel, "Attitudes towards Video and TV - UK - October 2019," Mintel, Tech. Rep., 2019.

[47] National Grid ESO. (2020). The 'lockdown effect' on TV viewing habits and the electricity grid, [Online]. Available: https : / / www . nationalgrideso. com / news / lockdown - effect - tv-viewing - habits - andelectricity-grid (visited on 04/09/2020).

[48] A. Kazi et al., "A survey of sitting time among UK employees," Occup. Med. (Lond.), vol. 64, no. 7, pp. 497-502, 2014.

[49] S. A. Clemes et al., "Sitting time and step counts in office workers," Occup. Med. (Lond.), vol. 64, no. 3, pp. 188-192, 2014

[50] S. A. Clemes, S. E. O' connell, and C. L. Edwardson, “Office workers' objectively measured sedentary behavior and physical activity during and outside working hours," J. Occup. Environ. Med., vol. 56, no. 3, pp. 298-303, 2014.

[51] S. Cassidy et al., "Cross-sectional study of diet, physical activity, television viewing and sleep duration in 233110 adults from the UK Biobank; the behavioural phenotype of cardiovascular disease and type 2 diabetes," BMJ Open, vol. 6, no. 3, e010038, 2016.

[52] H. Hamasaki, "Daily physical activity and type 2 diabetes: A review," World J. Diabetes, vol. 7, no. 12, p. 243, 2016.

[53] A. Fry et al., "Comparison of sociodemographic and healthrelated characteristics of UK Biobank participants with those 
of the general population," Am. J. Epidemiol., vol. 186, no. 9, pp. 1026-1034, 2017. 